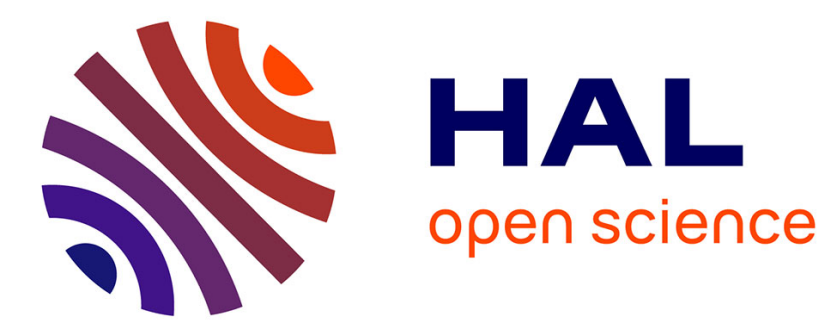

\title{
A Framework for Task-Based Ambidexterity in Manufacturing SMEs
}

Kristina Sollander, Annika Engström, Joakim Wikner

\section{To cite this version:}

Kristina Sollander, Annika Engström, Joakim Wikner. A Framework for Task-Based Ambidexterity in Manufacturing SMEs. IFIP International Conference on Advances in Production Management Systems (APMS), Aug 2018, Seoul, South Korea. pp.518-525, 10.1007/978-3-319-99704-9_63 . hal02164872

\section{HAL Id: hal-02164872 \\ https://hal.inria.fr/hal-02164872}

Submitted on 25 Jun 2019

HAL is a multi-disciplinary open access archive for the deposit and dissemination of scientific research documents, whether they are published or not. The documents may come from teaching and research institutions in France or abroad, or from public or private research centers.
L'archive ouverte pluridisciplinaire HAL, est destinée au dépôt et à la diffusion de documents scientifiques de niveau recherche, publiés ou non, émanant des établissements d'enseignement et de recherche français ou étrangers, des laboratoires publics ou privés. 


\title{
A framework for task-based ambidexterity in manufacturing SMEs
}

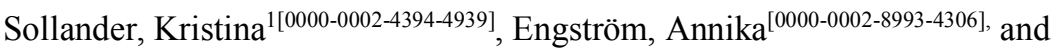 \\ Wikner, Joakim ${ }^{\text {[0000-0003-2252-5337] }}$ \\ Jönköping University, Jönköping, Sweden \\ ${ }^{1}$ kristina.sollander@ju.se
}

\begin{abstract}
To be competitive, it is important for companies to create a breeding ground for innovation without jeopardizing productivity. The challenge posed by industrial companies and the innovation research community is how to promote innovation while achieving efficient execution. The ability to balance execution and innovation is referred to as organizational ambidexterity (OA), which includes several dimensions, concepts and approaches where a central task can be identified. The purpose of this paper is to investigate what consequences result from different dimensions of a task in relation to the types of its actions, and their effects on OA. The focus of the task is firstly investigated, followed by the development of nine scenarios via combining the designers' and the performers' perspectives of the task. A brief analysis of the scenarios indicates that there is no single optimal scenario; rather, the scenarios represent different states that are appropriate for certain conditions, and dynamic adaptation should be encouraged in relation to the changing conditions. This type of dynamics is particularly expected to prevail in small and medium-sized enterprises (SME) because the roles that are responsible for tasks in these organizations are less specialized. Therefore, SMEs must define tasks that include both explorative and exploitative parts, either simultaneously or sequentially, to stimulate employees to work ambidextrously and thereby develop the concept of task-based ambidexterity.
\end{abstract}

Keywords: Organizational ambidexterity, Task-based, SME.

\section{Introduction}

An important contribution of the field of operations management is the strengthening of the manufacturing industry [1]. Competitiveness is challenging to establish; as shown by Porter [2], it can be either strictly focused on cost leadership or differentiation by other means. For a company to be competitive regarding differentiation by other means, it is of particular importance to create a good breeding ground for innovation. To support differentiation it is important that innovation embrace implementation of new and/or significantly improved processes and methods as well as develop new products for the market, however, a narrow focus regarding innovation can jeopardize productivity if it is not carefully employed [3]. Some ideas are not fruitful, and "trial 
and error" might require the use of several resources without significant results [4]. By contrast, the drive for efficiency might inhibit innovation [3], and lack of time, reduction of risk, and variation all affect the innovation culture negatively [5].

From this perspective, a workplace can be seen as a site of two logics [6] that are interdependent but often competing for resources. One logic is characterized by exploitation, the use of current knowledge to execute a task. The other logic is characterized by exploration, the development of new knowledge to innovate. Here, we face a dilemma. The challenge posed by industrial companies is how to promote innovation while achieving efficient execution. The ability to balance execution and innovation, which is referred to as organizational ambidexterity (OA), is essential for sustained growth and survival [7].

Current OA frameworks are primarily developed in larger multi-divisional organizations and typically based on split organizational designs, where execution-exploitation actions are relegated to established operations divisions, while innovation-exploration actions are mainly the responsibility of research \& development (R\&D) and new venture divisions. However, the organization of small- and medium-sized enterprises (SMEs) is not suitable for such a division because these organizations are usually based on a more integrative perspective. SMEs provide particular opportunities for investigating and extending the knowledge of OA because of the more integrative organizational design of SMEs. The need for OA research in manufacturing SMEs is consequently interpreted as a two-fold issue: SMEs have a strong potential to take advantage of adapting OA practices and the SME context provides interesting opportunities for capturing the OA-phenomenon.

A key characteristics of SMEs is their flat and flexible organizational structures which put more emphasis for their success, compared to larger organizations, on the CEO's knowledge and competence versus managerial practices and processes [8]. Furthermore, these organizations are also more dependent of the CEO's capability to innovate [9]. Recently, scholars have questioned whether small companies' dependence on the CEO's individual knowledge is effective or whether it actually underutilizes other employees' talents and knowledge [10]. Knowledge creation in SMEs is often based on experience, is tacit, and is generally strongly connected to a few individuals [11]. Their small organizational numbers mean that they rarely have the aforementioned split arrangements found in larger firms. It is typical for one employee to be responsible for several functions [10]. Within the context of manufacturing SMEs it is therefore relevant to consider both managers and other employees as developers of their own work [9]. Expertise, experience, ideas, creativity, and skills among employees are drivers as well as valuable resources regarding the company's innovative work [12,6]. A concept known as employee-driven innovation (EDI), which emphasizes the importance of the employees' contribution in innovation, regardless of their position in the company, has therefore been suggested [12]. From this perspective, new knowledge of ambidexterity can be developed from understanding how the phenomenon occurs within the SME context and thus supporting SMEs to benefit from the new knowledge on how to manage the balance between exploitation and exploration. 
The research area of OA includes several dimensions, concepts and approaches of ambidexterity. Structural ambidexterity is the most researched concept of ambidexterity in organizational structures, it deals with integrative structural mechanisms to achieve ambidexterity [7]. Contextual ambidexterity means that an organization shifts form exploitation to exploration according to the changing demands of its operating environment (its context) [13]. The concept of individual ambidexterity emphasizes an individual's ambidexterity versus that of the organization [14]. There are also sequential and simultaneous ambidexterity approaches, where the main difference is temporal [15]. The task is a central level of analysis for them all.

The purpose of this paper is to investigate what consequences result from different dimensions of a task in relation to the types of its actions, and their effects on OA. Furthermore, the paper outlines empirical studies to pursue in relation to manufacturing SMEs and the findings.

\section{Research method}

This conceptual paper stems from a research project about innovation capabilities within manufacturing SMEs, initiated in 2018, and up till this point no empirical data has been analyzed. The literature was collected through snowball sampling with the purpose of understanding the concepts, approaches, dimensions, and types of actions within OA from a task-based perspective.

\section{Theoretical framework}

The unit of analysis here is the task to be performed, which acts as a foundation for the introduced concepts, approaches, dimensions, and types of actions that are connected to OA. OA is firstly outlined, including the knowledge dimensions of exploration and exploitation. Then, the three concepts of $\mathrm{OA}$, structural, contextual, and individual, are described. Thereafter, the sequential and simultaneous approaches are presented. Finally, the designing and performing of the task's knowledge dimensions of execution and innovation are outlined.

\subsection{Dimensions of OA}

March [4] concluded that, to stimulate OA, an organization needs to adapt quickly to employees' ideas rather than vice versa. He developed two knowledge dimensions, exploration and exploitation, which he recommends should be balanced within organizations. His definitions of the terms are as follows: "Exploration includes things captured by terms, such as search, variation, risk-taking, experimentation, play, flexibility, discovery, and innovation. Exploitation includes such things as refinement, choice, production, efficiency, selection, implementation, and execution." Companies tend to focus their resources in exploitation mode [6,4]. A study by O'Reilly and Tushman [7] indicated that innovative projects proved to be more successful if they were based in an 
ambidextrous organization that had the ability to balance the exploration of new possibilities and the exploitation of old certainties. If the focus was on exploration without exploitation, the cost of experimentation would be high and often without benefit. If the opposite occur, and the focus was on exploitation without including exploration, there would be a high risk of facing a suboptimal stable equilibrium [4].

\subsection{Concepts of $\mathrm{OA}$}

The concepts of particular significance for OA are related to structural, contextual, and individual perspectives. Structural ambidexterity, the most researched concept, occurs when firms jointly conduct exploration and exploitation activities by developing specialized compartmental structures [16]. It could be viewed as structuring the organization's business units to achieve ambidexterity, where the business units are either independent or interdependent of each other [15]. Contextual ambidexterity is defined as "the behavioral capacity to simultaneously demonstrate alignment and adaptability across an entire business unit" [13]. All aspects of the organization are working together toward the same goal, and activities are adapted within the business unit to meet changing demands. Individual ambidexterity is defined as a human's cognitive ability to flexibly adapt by shifting between exploitation and exploration in a simultaneous or sequential cycle [14]. If the organizational context is dynamic and unpredictable, it is necessary to have individual ambidexterity to achieve success [17].

\subsection{Approaches of $\mathrm{OA}$}

The approaches of OA have either sequential or simultaneous character. In the sequential approach, the organization periodically switches between exploration and exploitation either within or between business units to become ambidextrous over time. It is believed that it is possible for a company to find focus and be efficient by being internally consistent [16]. The simultaneous approach is connected to adaptive systems research, and it is believed that successful businesses should be able to balance both explorative and exploitative tasks via either separated or united performers at once because they often reinforce one another. Some studies have indicated that organizations that balance ambidexterity simultaneously tend to be more innovative [16]. However, to ensure that business units align, overall values and strategies should be shared [7].

\subsection{Designing and performing tasks}

To stimulate employees' contributions, tasks can be designed by the organization to include important ingredients, such as the following: 1) variation to support the use of different skills, 2) opportunities to see the entire picture (i.e., the complete product realization process as well as employees gaining an understanding of the meaning of their work as part of a whole), 3) autonomy to influence the situation, 4) autonomy in the development of the task, and 5) feedback on performance [18]. More or less complex tasks cause different patterns of behavior and require different types of learning and knowledge- from routines to reflection [6]. 
A task consists of activities which is the sum of aggregated actions performed by employees and/or machines. An action stands for the smallest entity of work that an activity can be divided into [19]. An execution action involves the responsibility to follow instructions and procedures to control product quality. An innovation action involves the responsibility to develop and improve the nature of the task itself [6].

\section{OA from a task perspective}

To achieve OA, the task perspective is interesting to investigate since it offers an opportunity to study the interplay between the task designer and the task performer. As a foundation for task-based ambidexterity, the focus of the task is first established. Thereafter, the task is defined in terms of "design" and "perform", which, in combination, make it possible to identify nine scenarios of the task.

\subsection{Task focus}

One way of explaining a task's focus is through the knowledge dimensions, including exploration only, exploitation only, or both (Table 1). If there is no influence of neither exploration nor exploitation it is categorized as void based on these two dimensions (1). When only exploration is required, the focus of the task is to innovate (2). If only exploitation is required, the focus of the task is to execute (3). Finally, when the task requires both exploration and exploitation at the same time, both dimensions are needed to execute and innovate, which here is called exovate (4), meaning that simultaneous ambidexterity is required.

Table 1. The focus of the task, based on Engström and Wikner [12]

\begin{tabular}{l|c|c|}
\multicolumn{1}{c}{} & \multicolumn{1}{c}{ No Exploration } & \multicolumn{1}{c}{ Exploration } \\
\cline { 2 - 3 } No Exploitation & 1. Void & 2. Innovate \\
\cline { 2 - 3 } Exploitation & 3. Execute & 4. Exovate \\
\cline { 2 - 3 }
\end{tabular}

If sequential ambidexterity is applied, the task's focus switches between innovate (2) and execute (3); however, no exact estimation regarding how much time could pass between the two states of a task, and still refer to the ambidexterity as sequential, has been identified in the literature.

\subsection{Ambidexterity scenarios based on task design and task perform}

Task design can be conducted by either an organization or a manager, or by an employee without a formal management position. The task is designed in one of three different dimensions (Table 1, focuses 2-4). If the designer promotes innovation, it will encourage development through new ideas, questioning current work processes, and experimentation. If it promotes execution, it will encourage effectiveness, following routines, and adjusting current work processes. If the designer has expectations regarding exovation, it will encourage both innovation and execution simultaneously. 
Employees consequently perform tasks focusing on either execution, innovation, or exovation. Actions of execution are defined as exploitative, actions of innovation are defined as explorative and actions of exovation are defined as both, simultaneously. By combing the design and perform parts of a task, it is possible to identify nine scenarios, as shown in Table 2, where Exe indicates Execute, Inn indicates Innovate, and Exo indicates Exovate.

Table 2. Designing and performing a task

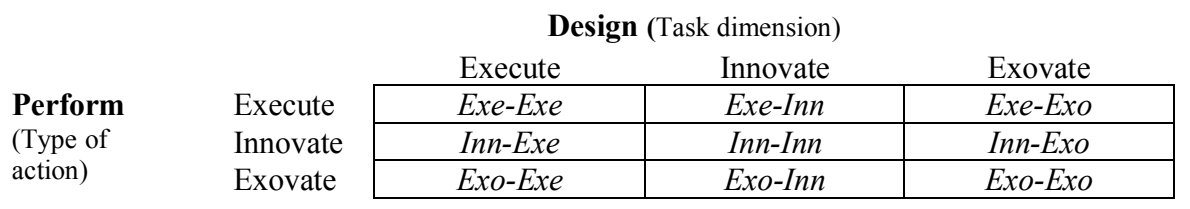

In Exe-Exe, the focus of both the designer and the performer is execution, which is characterized by short-term efficiency. Importantly, this might lead to suboptimal stable equilibrium if extended long term. In Inn-Inn, the focus is on innovation, which is likely conducive to growing ideas; however, if it is not based on applicability regarding execution, there is a great risk of implementing an expensive experiment that is either impossible or ineffective. In Exo-Exo, both parties design and perform the task simultaneously ambidextrous, which is likely a good environment for applying innovative ideas and streamlining operations. Exe-Inn and Inn-Exe are both interesting because their foci differ between the designer and the performer. In Exe-Inn, the focus of the designer is on innovation, while the performer stresses execution. One cause for this could be overly high expectations from the designer compared to the capability of the performer, which can cause frustration for both parties. In Inn-Exe, the designer's focus is execution, while the performer emphasizes innovation. One cause for this could be that the performer uses potential innovations that the designer is unable to capture. Although this could cause frustration and dejection within the company, it could potentially be solved by creating different forums for innovation. Notably, both Exe-Inn and Inn-Exe offer strong possibilities for development. In the scenarios of Exe-Exo and InnExo, exovation is encouraged by the designer but is performed as either execution or innovation. Further study is needed in this area; yet, it could be related to a combination of the same issues as in Exe-Inn and Inn-Exe (i.e., lack of performer competence and/or a designer who is unable to capture innovation). The scenarios of Exo-Exe and ExoInn, where the performer is exovate but the designer only requires execute or innovate, represent situations where performers are more capable than needed.

Interestingly, this brief analysis indicates that Exe-Exe, Exe-Inn, Inn-Exe, Inn-Inn and Exo-Exo all seem to have limitations in that each scenario is not sustainable in the long-term. All scenarios offer advantages and disadvantages but remaining in one scenario for an extended time will probably be costly in some form.

Moving between Exe-Exe, Inn-Inn, and Exo-Exo and utilizing ambidexterity in either a sequential or simultaneous way could potentially be more efficient from a longterm perspective. Scenarios in which the designer and the performer are aligned and share the same view of the task will more likely be fruitful, easier regarding resource allocation and finding the right competences, and better at avoiding frustration. Being 
in Exo-Exe and Exo-Inn could be better if the aim is either Exe-Exe, Inn-Inn, or ExoExo compared to being in Exe-Exo or Exe-Inn. This is because it is probably easier for the designer to consider if the task should be designed differently and, if so, to change it compared to changing either the competence or the culture of the performer(s).

\section{The concept of task-based ambidexterity}

By identifying and mentally visualize the concept of task-based ambidexterity we can identify the interplay between the designer and the performer, thus, finding a way to study the phenomenon of ambidexterity more thoroughly. EDI supports the idea of all employees' contributions to innovation; however, the concept of task-based ambidexterity outlined here highlights the importance of employees also focusing on exovation to create a balance between innovation and execution. This requires the organization to design tasks that include both explorative and exploitative parts, which are implemented either simultaneously or sequentially, to stimulate employees to work ambidextrously. This relates well to Hackman's [18] critical aspects of tasks that help employees stimulate knowledge creation in organizations and March's [4] statement that organizations need to adapt quicker to employees than vice versa.

Since the SME context is often both dynamic and unpredictable compared to the context of larger organizations, it is crucial for SMEs to stimulate their employees to be ambidextrous to achieve success. This indicates that SMEs could benefit from more knowledge about OA to make explicit decisions, moving between the different scenarios. Studying the context of SMEs can provide mutual benefits; such as insights into the concept of task-based ambidexterity together with benefits that SMEs can obtain.

This research clearly indicates that ambidexterity is more than a static mode; instead, it can be achieved in several ways both sequentially and simultaneously. Future research could examine the time perspective as well as how OA occurs on different organizational levels. Empirical studies could also be used to understand the interplay between the performer and the designer, including how employees perceive the task given by the designer and vice versa. By using either an in-depth or a narrative approach, it would be possible to gain a deeper understanding of the phenomenon.

Acknowledgements. This research was performed in collaboration with industry in project Innovate (KKS-20170312) and were financed by the research environment SPARK at Jönköping University and The Knowledge Foundation (www.kks.se)

\section{. References}

1. Slack, N., Chambers, S., Johnston, R.: Operations Management. Pearson Education, (2010)

2. Porter, M.E.: Competitive Advantage of Nations: Creating and Sustaining Superior Performance, vol. 2. Simon \& Schuster, (2011)

3. Lovén, E.: Kreativitet, innovation och lean. In: Sederblad, P. (ed.) Lean i arbetslivet. Liber, Stockholm (2013) 
4. March, J.G.: Exploration and exploitation in organizational learning. Organization Science 2(1), 71-87 (1991).

5. Hongyi, C., Taylor, R.: Exploring the impact of lean management on innovation capability. In: Management of Engineering \& Technology, 2009. PICMET 2009. , Portland 2009, pp. 826-834. IEEE

6. Ellström, P.-E.: Two logics of learning. In: Antaqnacopoulou, H., P, J., Andersen, V., Elkjaer, B., Hoyrup, S. (eds.) Learning, Working and Living. Mapping the terrain of Working Life Learning. Palgrave Macmillan, London (2006)

7. O'Reilly, C.A., Tushman, M.L.: Organizational ambidexterity: Past, present, and future. The Academy of Management Perspectives 27(4), 324-338 (2013).

8. Smith, M.H., Smith, D.: Implementing strategically aligned performance measurement in small firms. International Journal of Production Economics 106(2), 393-408 (2007).

9. Burton, M.D.: The company they keep: Founders' models for organizing new firms. Articles \& Chapters, 259 (2001).

10. Karltun, J.: On stage. Acting for development of businesses and ergonomics in woodworking SMEs. Linköping University (2007)

11. Ates, A., Garengo, P., Cocca, P., Bititci, U.: The development of SME managerial practice for effective performance management. Journal of Small Business and Enterprise Development 20(1), 28-54 (2013).

12. Høyrup, S., Bonnafous-Boucher, M., Hasse, C., Lotz, M., Møller, K. (eds.): EmployeeDriven Innovation. A New Approach. Palgrave Macmillan, Hampshire (2012)

13. Gibson, C.B., Birkinshaw, J.: The antecedents, consequences, and mediating role of organizational ambidexterity. Academy of Management Journal 47(2), 209-226 (2004).

14. Good, D., Michel, E.J.: Individual ambidexterity: Exploring and exploiting in dynamic contexts. The Journal of Psychology 147(5), 435-453 (2013). doi:10.1080/00223980.2012.710663

15. Simsek, Z.: Organizational ambidexterity: Towards a multilevel understanding. Journal of Management Studies 46(4), 597-624 (2009).

16. Chen, E.L., Katila, R.: Rival interpretations of balancing exploration and exploitation: Simultaneous or sequential. Handbook of Technology and Innovation Management, 197-214 (2008).

17. Davis, J., Eisenhardt, K., Bingham, C.: Optimal structure, market dynamism, and the strategy of simple rules. Administrative Science Quarterly 54, 413-452 (2009).

18. Hackman, J.R., Oldham, G.R.: Word Redesign. Addison-Wesley Publishing Company Inc, Massachusetts (1980)

19. Engström, A., Wikner, J.: Identifying scenarios for ambidextrous learning in a decoupling thinking context. In: Lödding, H., Riedel, R., Thoben, K.-D., von Cieminski, G., Kiritsis, D. (eds.) Advances in Production Management Systems. The Path to Intelligent, Collaborative and Sustainable Manufacturing : IFIP WG 5.7 International Conference, APMS 2017, Proceedings, Part II. pp. 320-327. Springer International Publishing, Cham (2017) 